\title{
INTERPRETATION OF RADIO-ECHO RETURNS FROM INTERNAL WATER BODIES IN VARIEGATED GLACIER, ALASKA, U.S.A.
}

\author{
By Robert W. Jacobel and Stefan K. Anderson
}

(St. Olaf College, Northfield, Minnesota 55057, U.S.A.)

\begin{abstract}
Radio echo-sounding studies were used to detect water-filled cavities on Variegated Glacier, Alaska, during its surge in 1983. Cavity locations were determined by spatial surveys, and changes in cavity size and water content over a 5 week period were inferred from phase and amplitude changes in the echo wave form. Data were taken at both 4 and $8 \mathrm{MHz}$ central frequencies of the impulse transmitter. These bi-frequency results, together with spectral analysis of the digitized wave forms, were used to provide additional information about the cavity dimensions. Cavity response is interpreted in terms of a simplified model of a thin layer of strong dielectric contrast. Computer simulations of radar pulses encountering such layers reproduce the main features of the data.
\end{abstract}

\section{INTRODUCTION}

Radio echo-sounding studies on Variegated Glacier, Alaska, during the surge-initiation phase in 1982 showed internal echoes in the upper $100 \mathrm{~m}$ whose intensity varied considerably over time-scales of days to weeks (Jacobel and Raymond, 1984). Recorded during times of high basal water pressures, these echoes were interpreted as arising from decimeter-sized water-filled cavities which emptied and filled primarily in response to the input of surface water and not to changes in basal water pressure. As such, they were inferred to have considerable hydraulic resistance to the bed. Information on the size of the cavities was derived from spectral analysis of the echoes which showed a strongly peaked power spectrum in contrast to the broadband characteristics of the impulse wave form. Because the system was fixed on the glacier surface, however, the precise location of the cavities could not be calculated, although their distance from the receiver and approximate depth were determined.

In 1983, radio echo-sounding studies were conducted during May and June while the surge was in progress and peak velocities were occurring (Kamb and others, 1985). The location of the apparatus at $\mathrm{Km} 9.5$ near the equilibrium line was approximately the same as in the previous year (Fig. 1). In these studies, however, the transmitter was movable, enabling us to conduct spatial surveys to locate the positions of internal echoes. In addition, the center frequency of the impulse radar could be selected to be either 4 or $8 \mathrm{MHz}$ (approximately) by changing the antenna length, thus giving information on the response of the cavities to radiation at two center frequencies.

The radar transmitter, which produces an impulse with broad-band characteristics, has been described previously (Watts and England, 1976; Watts and Wright, 1981). The recording system utilized a sampling oscilloscope to receive the returned wave form, and analog information was recorded on tape and digitized off-line. This is the same system used on Variegated Glacier in 1982, and has been described in detail by Jacobel and Raymond (1984). Improvements in the off-line processing now enable us to display digitized wave forms in adjacent columns, as is done in seismic profiles. For radar wave forms, this allows a comparison of phase as well as amplitude information.
ECHO WAVE FORMS, CAVITY LOCATIONS, AND CHANGES IN TIME

Figure 2 shows data recorded from 13 May to 16 June 1983 with the antennae oriented parallel to the south-west glacier margin in the configuration marked (a) in the inset of Figure 1. The first three wave forms were recorded over a $12 \mathrm{~d}$ period, and thereafter each one depicts a daily recording. Echo time, indicated in the right margin, has been converted to a distance shown along the left margin using the center point between the transmitter and receiver as the origin of coordinates and a wave speed of $168 \mathrm{~m} \mathrm{~s}^{-1}$. Wave amplitudes from greater distances have been corrected for attenuation by the scaling function shown just inside the left margin. This scaling also has the effect of suppressing the direct air-wave arrival which was used as the system trigger.

A number of interesting features are apparent in these data. The most prominent is the echo near 2200 ns which, except for the final two records, is seen to increase in amplitude uniformly throughout the time period while moving to a slightly greater distance from the radar apparatus. Gradual changes occur in the phase of this echo, particularly in the first and last weeks of the period covered.

A second pronounced echo at approximately $700 \mathrm{~ns}$ also grows and then slightly decreases in amplitude in a uniform manner. However, this one undergoes a more obvious change in phase which is most noticeable after 1 June. A less-pronounced echo is observable at approximately $1400 \mathrm{~ns}$ which also uniformly increases and then decreases in amplitude while undergoing a change in phase most noticeable after 5 June.

In the echo wave forms from the three reflectors neither amplitude nor phase change coincidently. This is strong evidence that the observed effects are not due to system changes and, also more importantly, that they are not due to changes in the medium - effects produced in the snow-pack or the shallow firn.

Before proceeding further with a discussion of changes in these echoes, we next present information about the spatial locations of the reflectors. Several times during the recording period the radar transmitter was moved temporarily to different sites and wave forms were recorded by the fixed receiver. Although a dipole antenna is not highly directional, very little energy is transmitted or can be received along the antenna axis, whereas the maximum amount of energy is transferred when antenna axes are parallel. Thus, directional information on the location of echoes can be obtained.

By moving the transmitter at intervals in a circle about the receiver, it was determined that the strongest echoes discussed above were located approximately south-west of the receiver, toward the glacier margin. A cross-glacier profile was therefore run in that direction and eight transmitter positions were established at $5 \mathrm{~m}$ intervals along the cross-glacier line south-westward from the receiver. In all cases the dipole antennae were parallel to each other and 


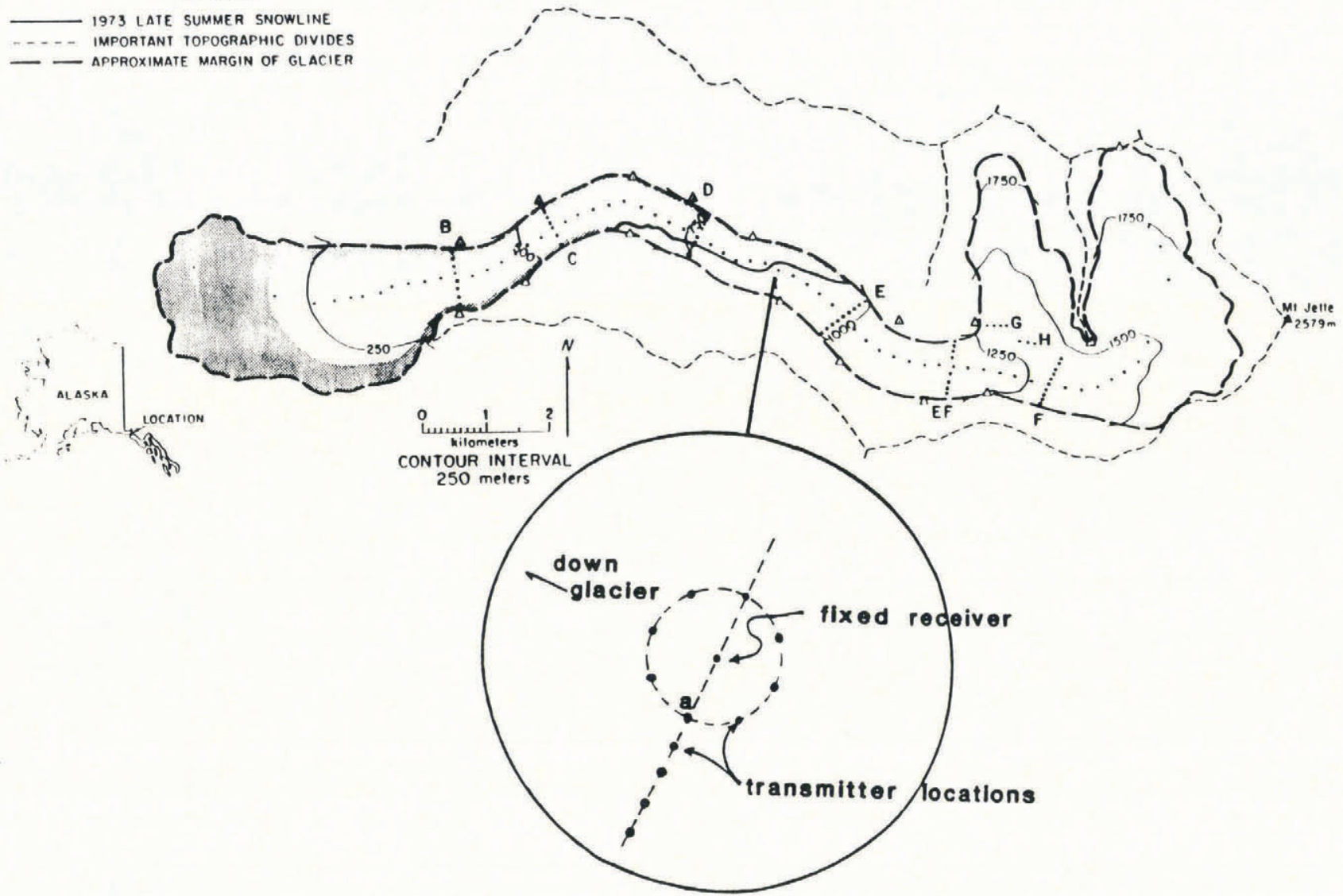

Fig. 1. Location map of Variegated Glacier with inset showing position of fixed radar receiver and typical transmitter locations in the spatial surveys. The transmitter position marked (a) on the circle south-west of the receiver was used to acquire the data in Figure 2.

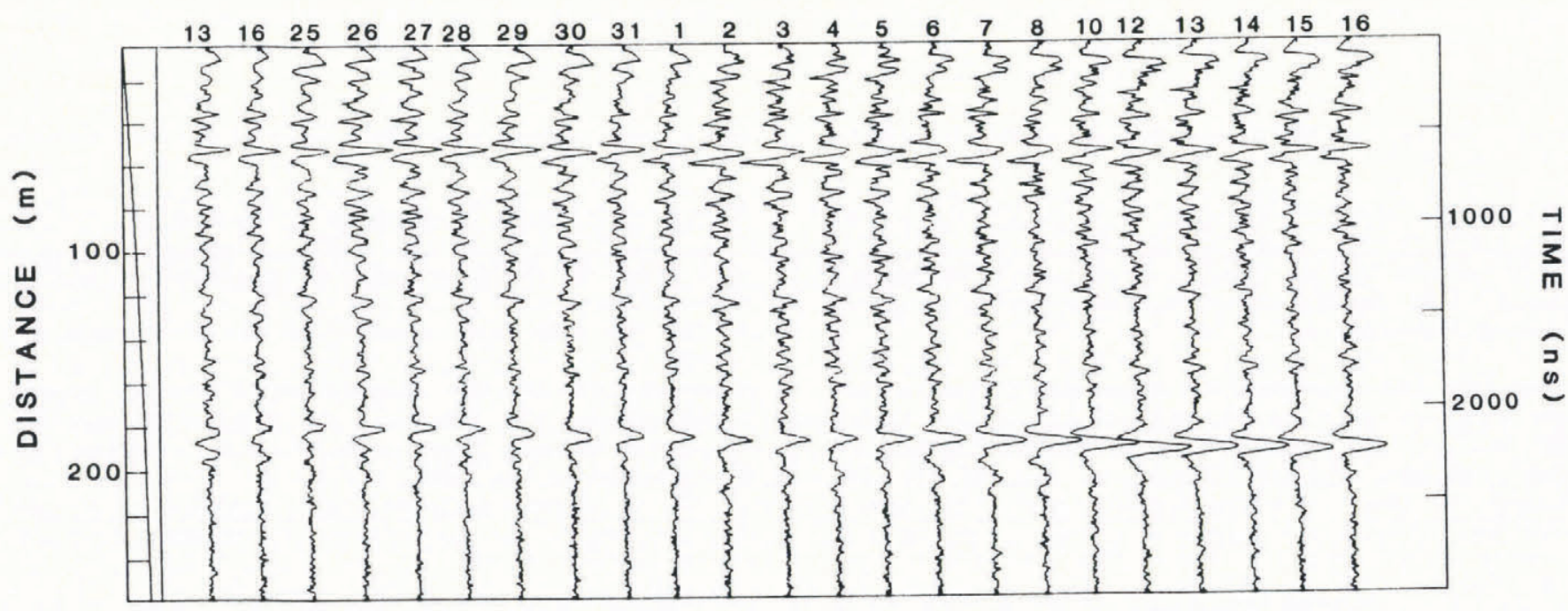

Fig. 2. Radar wave forms from 13 May to 16 June 1983, showing echoes from internal water bodies at approximately 2200,1400, and $700 \mathrm{~ns}$. Time origin is the arrival of the direct wave.

thus were approximately parallel to the glacier center line (Fig. 1).

Figure 3 shows results from one such profile study made on 26 May. The figure is an approximately north-east-south-west cross-sectional profile of the glacier with the receiver and region of transmitter locations indicated on the surface. The glacier bed has been estimated from Bindschadler and others (1977). Each of the three echoes discussed above was located in the wave forms recorded from the eight transmitter positions shown. In this cross-section, the locations of all reflectors are constrained to lie along ellipses with transmitter and receiver at the foci, and the equations for these ellipses were solved simultaneously for the echo coordinates of the reflectors. Plotting symbols in the figure represent simultaneous solutions to the equations for pairs of transmitter positions. 


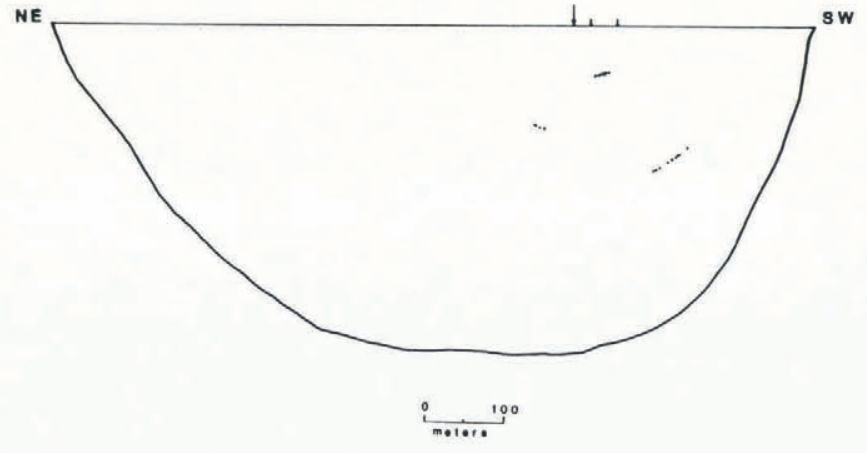

Fig. 3. Results of cross-glacier spatial survey of 26 May to locate the positions of reflectors producing the echoes seen in Figure 2. Arrow and marks on the surface indicate the fixed receiver position and the range of transmitter locations along the north-east-south-west profile.

It should be noted that, although the echo locations are assumed to lie in a plane trending generally south-west of the radar receiver, the spatial surveys do not precisely determine positions in three dimensions. The actual echo locations may lie somewhat up- or down-glacier from the cross-section depicted, but approximately at the depths and distances indicated. This uncertainty in the up-down-glacier direction leads to a spread in the two-dimensional solution set as may be seen in particular for the deepest echo. Uncertainties are also due to imprecise knowledge of the exact transmitter positions, and this effect is indicated approximately by the size of the plotting symbol. Because of the close spacing of the transmitter positions relative to the depths sounded, the simultaneous solutions are highly sensitive to the transmitter-transmitter separations, and for this reason combinations involving two adjacent transmitter positions have been excluded from the figure.

\section{MODEL RESULTS AND INTERPRETATIONS}

The echoes are seen to arise from three discrete sources within the ice that have strong dielectric contrast with the surrounding medium. Because of their location, signal strength, and time-dependent behavior, we identify these echoes as arising from water-filled cavities within the ice. Air-filled voids such as crevasses, or rock debris, are far less reflective than water (Jacobel and Raymond, 1984) and in any case are not likely to be found in these locations. Rock debris, further, would be unlikely to show echo variations with time.

In order to understand better the phase and amplitude changes seen in these echoes over time, we have calculated the response of an idealized water-filled cavity to electromagnetic radiation of various frequencies, and have also modeled its response to a broad-band pulse corresponding to our transmitted wave form. In the simplest case, a cavity may be approximated as a layer of some dielectric contrast illuminated with normally incident electromagnetic radiation. Multiple reflections and transmissions from the interfaces are accounted for in the calculation, but the shape and end effects of a real cavity are ignored in the planar approximation. Although real cavities are unlikely to be either planar or spherical, these are two geometries which can be treated simply, and models employing each of these have been successful in the interpretation of radar results (Watts and England, 1976; Jacobel and Raymond, 1984).

Figure 4 shows the amplitude and phase relative to the transmitted wave for monochromatic energy of wavelength $\lambda$ reflected normally from a water layer (index 9.0) in ice (index 1.78). This is plotted as a function of layer thickness expressed as a fraction of the wavelength in water, and so is independent of actual cavity size. The most striking feature is a result recognizable from elementary wavepropagation theory - the strong dependence of the reflection coefficient on the cavity thickness. The calculation shows near total reflection (constructive interference) for a range of cavity dimensions approximately equal to a quarter of a wavelength, and a reversal in phase. A similar result, calculated in terms of attenuation of the returned power, was obtained for a melt-water layer on the ice surface (Smith and Evans, 1972). For the case of an impulse consisting of a range of frequencies, such a layer could act as a filter, returning strongly those components with wavelengths near four times the cavity thickness and causing a "ringing" in the echo wave form. This was our interpretation of echoes seen in the 1982 Variegated Glacier radar data where $0.5 \mathrm{~m}$ sized cavities were identified on the basis of a strongly peaked power spectrum of the returned energy (Jacobel and Raymond, 1984).

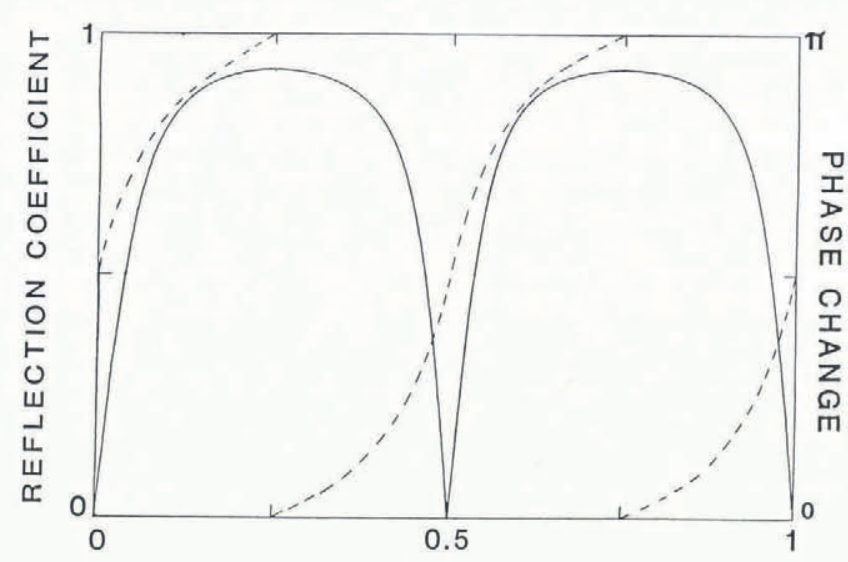

LAYER THICKNESS / WAVELENGTH

Fig. 4. Calculated amplitude (solid line) and phase (dotted line) of a monochromatic wave reflected from a water layer as a function of the thickness : wavelength ratio.

The figure also shows that a dielectric layer can act as a filter which will transmit an even narrower range of wavelengths at twice the layer thickness (constructive interference for the transmitted wave). For example, a watersoaked layer at the surface, in addition to being transparent to long wavelengths, will pass a narrow window of energy at a wavelength equal to twice the layer thickness. For a broad-band input impulse, it is possible that such a layer could also bias the power spectrum of received echo energy by removing all but a narrow band of frequencies.

Model results of the response of a dielectric layer to our broad-band impulse wave forms show qualitatively the filtering effects just described (see below). However, since most of the energy in the input impulse is centered around $8 \mathrm{MHz}$, layer widths must be made unrealistically large (on the order of several meters) in order for the filtering effects to dominate in the echoes. In order to see frequency filtering from more realistic decimeter- to meter-sized layers, most of the power in the input impulse which is at long wavelengths must be otherwise attenuated. In a real glacier this could conceivably occur as these longer wavelengths penetrate to deeper ice. The power spectrum of returned energy from internal reflectors will then be dominated by short-wavelength contributions from scattering, and will show a "blue bias" (Jacobel and Raymond, 1984).

This characteristic preponderance of higher-frequency components typically seen in the returned wave form from internal echoes will also arise from simple scattering as can be seen in Figure 4. For laminar cavities whose dimensions normal to the incident radiation are less than about 0.2 times the wavelength $(d / \lambda<0.2)$, the reflection coefficient is inversely proportional to the wavelength, or is proportional to the frequency. Thus, thin cavities tend preferentially to scatter and return the higher-frequency components of an input impulse - a familiar result from standard scattering theory. A similar calculation for spherical 
scatterers was shown by Watts and England (1976); frequency-dependent scattering is the reason that lowfrequency radars are necessary for detecting bedrock echoes from deep temperate ice.

In the present study, the three cavities discussed all return a single cycle of the incident wave, although each shows a different phase relative to it, and each has a somewhat different frequency content. Thus, we are not seeing cavities whose dimensions cause them to resonate at a single frequency present in the incident pulse. Instead, these are smaller structures whose dimensions normal to the radar, though perhaps differing somewhat from each other, are substantially less than the wavelengths predominantly present in the pulse incident upon them, i.e. less than $2 \mathrm{~m}$. Thus, they act like scattering sources, preferentially returning energy from the pulse at shorter wavelengths. The echo wave form therefore has a higher-frequency spectrum than the incident pulse, but still displays broad-band characteristics.

Differences between echo phases and pulse widths, and their changes over time are indicative of different cavity sizes and changes in them. This behavior is modeled in our simulations of an impulse incident on a dielectric layer, which show the phase, amplitude, and spectral content of the returned energy to be strongly dependent on cavity thickness. Figure 5 displays one example of this. The wave form at the top is the actual incident impulse from the radar transmitter with a central frequency of $8 \mathrm{MHz}$. Each of the succeeding wave forms is the model result of an echo from a cavity of greater width, starting with $0.1 \mathrm{~m}$ and doubling in each step to $12.8 \mathrm{~m}$. Amplitudes of the echoes increase with cavity thickness up to a maximum at about $1 \mathrm{~m}$ when the layer is one-fourth the wavelength in water of a wave at the central frequency. Thereafter, amplitude decreases slowly as the layer becomes thicker. The narrow layer changes the phase of the pulse by approximately $\pi / 2$. Thereafter, successively thicker layers cause the phase shift to approach $\pi$. This is what would be expected for a very thick layer - a single ice/water

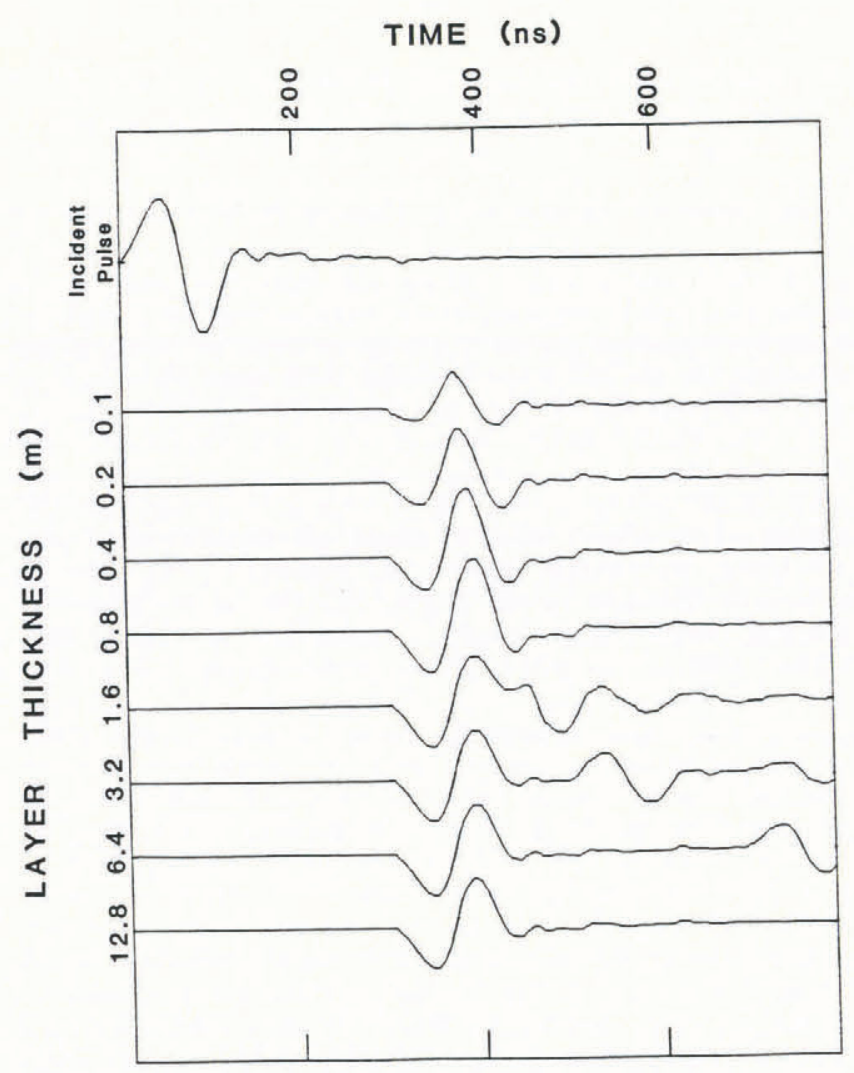

Fig. 5. Model results of a radar impulse, incident on a layer-like cavity of varying thickness showing changes in both phase and amplitude of the reflected wave form as the cavity thickness. boundary. Also evident in the figure is the ringing in the echo from multiple reflections which occur when the layer strongly returns or removes principal wavelength components which are present in the input impulse - in this case, when the layer is between $1.6 \mathrm{~m}$ and about $6.4 \mathrm{~m}$ thick.

The changes in amplitude and phase for the echo at 2200 ns shown in Figure 2 are broadly similar to the changes shown by the model for a layer which is increasing in thickness in the range where $d / \lambda$ varies from 0 to about 0.2 . In both cases, amplitude increases as the phase difference advances toward $\pi$. Phase difference in Figure 2 is indicated by the change in echo-pulse shape from the incident impulse (approximately a sinusoid) shown in Figure 4. This model result supports the interpretation of a cavity growing in size or filling with water from decimeter to near-meter thickness. The cavity at $700 \mathrm{~ns}$ behaves generally in a similar manner, although there appears to be a reversal in the trend during the last week, indicating that the cavity may be closing slightly.

Additional information about cavity size is provided by several data runs taken at a central frequency of $4 \mathrm{MHz}$ instead of $8 \mathrm{MHz}$. Figure 6 shows a comparison of 4 and $8 \mathrm{MHz}$ signals recorded on 2 June. The deepest cavity at $2200 \mathrm{~ns}$ is clearly seen in the same location in both data sets. The frequency characteristics of this echo in the $4 \mathrm{MHz}$ data correspond to the lower center frequency of the input impulse, and thus it appears wider and slightly shifted in time. The shallower cavities are not clearly seen in the $4 \mathrm{MHz}$ data, presumably because they are small enough to scatter poorly energy incident at these longer wavelengths. Energy returned at all depths in the $4 \mathrm{MHz}$ data is generally greater because of better coupling to the ice with the longer antennae. Echoes from deeper structures are further enhanced because less energy is lost due to scattering from smaller cavities. As an example, a reflector that can be seen clearly at approximately $3200 \mathrm{~ns}$ in the $4 \mathrm{MHz}$ data is only barely discernible above the noise in the $8 \mathrm{MHz}$ run.

A comparison of the phase relationship between the two echoes from the cavity at $2200 \mathrm{~ns}$ can be used to place rough constraints on its size. A $4 \mathrm{MHz}$ central frequency corresponds to a wavelength in water of about $8 \mathrm{~m}$. To be seen at all, the cavity must be larger than about $5 \%$ of this dimension in thickness or the pulse would simply pass through without reflecting appreciable energy. This results from simple scattering theory and is therefore essentially independent of the cavity shape assumed. Thus, a lower limit of $0.4 \mathrm{~m}$ seems reasonable for the cavity thickness. On the other hand, the phase of the echoes at both frequencies is similar, and corresponds most closely to the model case where $d / \lambda$ is less than about 0.2 . Here, the constraint comes from the $8 \mathrm{MHz}$ data which require that the cavity width be less than about $0.8 \mathrm{~m}$ on this date. These results are consistent with the size interpretations made on the basis of phase shifts in Figure 2, as described previously.

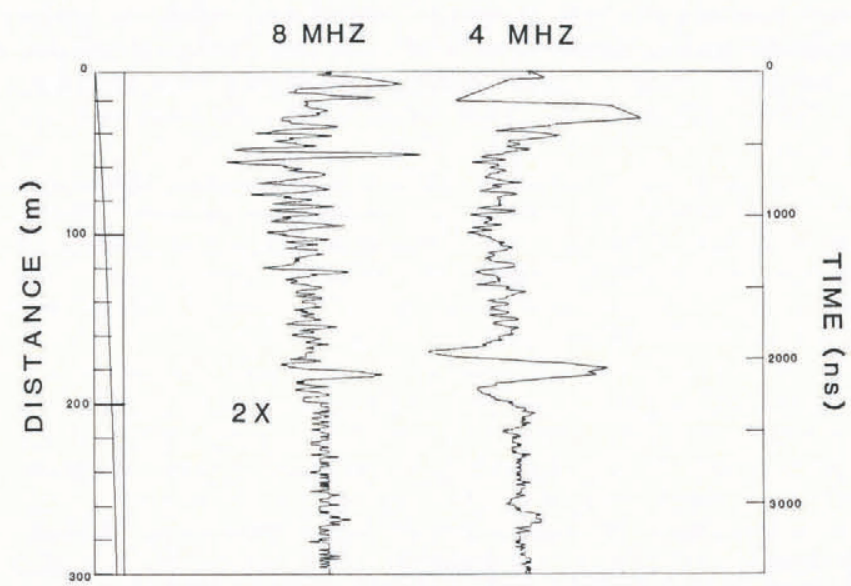

Fig. 6. Comparison of echo wave forms using $4 \mathrm{MHz}$ and $8 \mathrm{MHz}$ central frequencies for the transmitted impulse. 


\section{GLACIOLOGICAL IMPLICATIONS AND CONCLUSIONS}

It is difficult to relate the significance of these cavities and their changes in time in any simple way to the surge of Variegated Glacier given the limitations of the present data set. The focus here has therefore been on applying simple principles of physics to the echo interpretation - a necessary step to the more desired end. The hypothesis that such cavities exist is re-stated throughout the glaciological literature, so it is reasonable that remote-sensing techniques are beginning to reveal more about their presence. However, further studies are needed to understand more fully the real geometry of such cavities and the ways in which they evolve over time.

In the present case, the location and changes with time in the dimensions of these cavities are not related in a simple way to other glaciological variables that were measured, with the possible exception of ablation. During the latter part of the period, basal water pressure was measured in a bore hole approximately $200 \mathrm{~m}$ down-glacier from the radar apparatus. Basal pressure levels remained high and roughly constant near 4 bar below the overburden, corresponding to a water level in the bore hole $80 \mathrm{~m}$ below the surface (Kamb and others, 1985). Minor changes in these levels were not reflected in the radar records. This is similar to the situation which obtained in 1982 when borehole water-pressure levels were not correlated with changes in the strength of internal radar echoes. Throughout the period covered by the radar record, velocities in this part of Variegated Glacier were in excess of $12 \mathrm{~m} \mathrm{~d}^{-1}$, and inclinometer measurements indicated that most of the motion was due to basal slip (Kamb and others, 1985). Thus, in spite of the high velocities of the surge motion, this kind of plug flow would not be expected to produce stresses in internal cavities at the depths and locations indicated which would cause them to close rapidly.

As was the case in 1982, cavity changes seemed to correlate most strongly with surface input from ablation. For the first 2 weeks of the period covered, until approximately 26 May, total ablation was less than $20 \mathrm{~cm}$ of snow. New precipitation was in the form of snow and resulted in a net accumulation. In the latter 3 weeks, ablation increased rapidly totaling more than $1 \mathrm{~m}$, and new precipitation was primarily rain. The most noticeable changes in the radar record likewise occur after the third week in May when input from the surface increased significantly.

The most straightforward interpretation of these circumstances is consistent with the results from 1982; these cavities, which are located at depths between approximately 60 and $200 \mathrm{~m}$, have decimeter to meter dimensions perpendicular to the ray path. They enlarge (and possibly close) on time-scales of weeks, primarily in response to input from the surface. Lack of correspondence in changes of the echoes with changes in basal water pressures indicates that the cavities maintain considerable hydraulic resistance to the bed, and therefore probably play little direct role in controling the surge behavior other than acting as temporary storage reservoirs for water eventually delivered to the bed.

\section{ACKNOWLEDGEMENTS}

This work was supported by the National Science Foundation under grants DPP-8200725 and DPP-8406672. The field equipment for the radio echo-sounder was generously lent to us by the U.S. Geological Survey - Project Office Glaciology. The field work on Variegated Glacier was done while $R$. Jacobel was on a sabbatical at the University of Washington, and he wishes to thank in particular C. Raymond and others in the glaciology group for their support during that time. Several days of radar data were recorded by P. Mullen, E. Mezger, and K. Echelmeyer, of the California Institute of Technology, who also deserve thanks for their efforts. The radar data were digitized and processed at St. Olaf College by several under- graduate students in addition to S. Anderson. We both express our appreciation to them and to D. Olson of the Physics Department for his supervision of the interface development. Finally, we wish to acknowledge the thoughtful comments of one of the reviewers, whose careful reading and suggestions made parts of the manuscript more precise, thorough, and clear.

\section{REFERENCES}

Bindschadler, R.A., and others. 1977. Geometry and dynamics of a surge-type glacier, by R.A. Bindschadler, W.D. Harrison, C.F. Raymond, and R. Crosson. Journal of Glaciology, Vol. 18, No. 79, p. 181-94.

Kamb, B., and others. 1985. Glacier surge mechanism: 1982-1983 surge of Variegated Glacier, Alaska, by B. Kamb [and 7 others]. Science, Vol. 227, No. 4686, p. 469-79.

Jacobel, R.W., and Raymond, C.F. 1984. Radio echo-sounding studies of englacial water movement in Variegated Glacier, Alaska. Journal of Glaciology, Vol. 30, No. 104 , p. 22-29.

Smith, B.M.E., and Evans, S. 1972. Radio echo sounding: absorption and scattering by water inclusions and ice lenses. Journal of Glaciology, Vol. 11, No. 61, p. 133-46.

Watts, R.D., and England, A.W. 1976. Radio-echo sounding of temperate glaciers: ice properties and sounder design criteria. Journal of Glaciology, Vol. 17, No. 75, p. 39-48.

Watts, R.D., and Wright, D.L. 1981. Systems for measuring thickness of temperate and polar ice from the ground or from the air. Journal of Glaciology, Vol. 27, No. 97, p. 459-69. 